\title{
Model-Driven Harmonic Parameterization of the Cortical Surface
}

\author{
Guillaume Auzias ${ }^{1}$, Julien Lefèvre ${ }^{1,2}$, Arnaud Le Troter ${ }^{1}$, Clara Fischer ${ }^{3}$, \\ Matthieu Perrot ${ }^{3}$, Jean Régis ${ }^{4}$, and Olivier Coulon ${ }^{1}$ \\ ${ }^{1}$ LSIS Lab, UMR CNRS 6168, Marseille, France \\ ${ }^{2}$ Université de la Méditerranée, Marseille, France \\ ${ }^{3}$ Neurospin center, CEA, Gif-sur-Yvette, France \\ ${ }^{4}$ INSERM U751, Marseille, France
}

\begin{abstract}
In the context of inter-subject brain surface matching, we present a parameterization of the cortical surface constrained by a model of cortical organization. The parameterization is defined via an harmonic mapping of each hemisphere surface to a rectangular planar domain that integrates a representation of the model. As opposed to previous conformal mapping methods we do not match folds between individuals but instead optimize the fit between cortical sulci and specific iso-coordinate axis in the model. Experiments on both hemispheres of 34 subjects are presented and results are very promising.
\end{abstract}

Keywords: Surface matching, harmonic mapping, cortical organization, parameterization.

\section{Introduction}

The rise of surface-based methods for neuroimaging data analysis in the past 10 years has brought a new range of methods in the area of multi-subject comparisons: inter-subject surface matching. Such methods allow to define a common referential in which to perform group studies and inter-subject comparisons. The difficulty comes from the fact that, contrary to volume-based methods, the domain on which the data is defined is different from one subject to another. If one wants to register geometrical information or anatomical landmarks on the cortical surface between different subjects it is necessary to define a common domain on which to perform the registration. This is why existing methods map cortical surfaces to a standard geometry such as a sphere before registration. This mapping is either isometric or conformal [5]. Registration after or during the mapping generally optimize the matching of geometrical information (e.g. convexity [14]) or anatomical landmarks [1012138] but rarely embeds explicit information about the nature of the variability or stability of the cortical anatomy across subjects.

Nevertheless, the idea of an intrinsic organization of the cortical surface around which variability occurs has arisen [134], and it has been shown that a model of such organization can be used to perform cortical localization and implicitly 
solve the problem of inter-subject cortical surface matching [32. In particular in 2. a model is presented that embeds the concept of stable sulcal landmarks conjointly with the notion of organization of these landmarks through specific hypothesis of orientation and alignment of sulci. In this method, the authors propose a scheme of cortical organization, stating that a set of folds that correspond to the first folding location during antenatal life are stable across individuals, and that these folds are organized according to two orthogonal directions and two poles, the insular pole and the cingular pole (Fig:1). This model defines a natural spherical parameterization of the cortical surface, and in the same paper the authors propose an implementation of this model that computes a parameterization constrained by the model for any given cortical mesh and therefore provide a cortical localization and an implicit inter-subject matching. Essentially, this leads to a coordinate system in which the folds that are part of the model always have the same coordinate (longitude or latitude, specified by the model, Fig.11), with a smooth interpolation of the coordinate fields between the constraints. The drawbacks of this approach are that the two coordinate fields are computed independently, the method is therefore unable to theoretically guarantee the integrity of the coordinate system, and that there is no control over the isometric or conformal properties of the resulting mapping.

In this paper we integrate the assumptions given by model presented in [2] and propose a new method for mapping this model onto an individual cortical surface. The relevance of the anatomical hypothesis underlying the model will be investigated in future work. Indeed, we define a mapping that explicitly minimizes angular distortions while matching cortical folds with the model, and introduces a coupling of the two coordinate fields. The originality compared to previous similar harmonic mapping methods (e.g. [10]12]) lies in the fact that folds are not matched to a specific target (e.g. the same fold across subjects) but instead are matched to an iso-coordinate axis of the $2 \mathrm{D}$ coordinate system.

In the next section we present our method, and in section 3 the results of our algorithm applied to a set of subjects are presented and discussed.

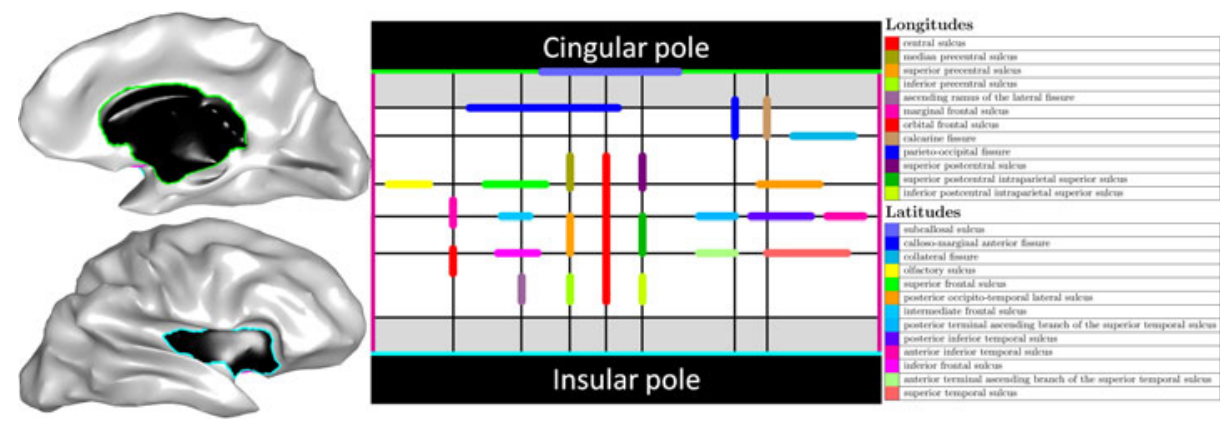

Fig. 1. Left: insular and cingular poles shown on an inflated hemisphere. Right: flat representation of the sulcus-based model of cortical organization. 


\section{Method}

We detail here our method to define a parameterization of the cortical mesh under the sulcal constraints defined on Fig 1 We formalize the parameterization of the cortex as follows. Let us denote $S_{T} \in \mathbf{R}^{3}$ the cortical mesh. The parameterization of $S_{T}$ consists in finding a suitable domain $\Omega \subset \mathbf{R}^{2}$ and a piecewise linear mapping $h: S_{T} \rightarrow \Omega$ that is linear on each triangle in $S_{T}$ and continuous. Such a mapping is uniquely determined by the images $u=\left(u_{1}, u_{2}\right)=h(v)=\left(h_{1}\left(v_{1}, v_{2}, v_{3}\right), h_{2}\left(v_{1}, v_{2}, v_{3}\right)\right) \in \mathbf{R}^{2}$.

The first step is the definition of the parameterization domain, presented in the next section.

\subsection{Parameterization Domain}

In contrast with previous approaches, the geometrical assumptions given by our anatomical model impose some constraints on sulcal landmarks but also on the insular and cingular poles, the later being defined around the corpus callosum. While the cortex can be represented as a surface which topology is spherical, i.e. closed without self intersection, the tessellation corresponding to an hemisphere remains open as a hole is formed by the cut around the corpus callosum, i.e. the cingular pole. When a parameterization is achieved on a spherical domain, this hole can be artificially closed but the closing influences the resulting coordinate system. We alternatively suggest to consider the open hemi-cortical representation for fitting our model.

The hemi-spherical cortical surface can then be subdivided into 3 anatomical patches : the insular pole, the cingular pole and the rest of the neocortex. The cingular pole, i.e. the corpus callosum, does not need to be parameterized, as it is not cortex. The parameterization of the insular pole is quite straightforward as its geometry is very close to a plane and is not detailed in the present contribution. Both poles can be segmented automatically (e.g. as presented in [2]), so we focus on the parameterization of the rest of the cortex which is the most challenging.

At this point, the neocortex is represented as a surface with two holes that has the same topology as a cylinder (Fig (2). A cut in the surface linking the two poles is mandatory to obtain the desired topology. We suggest to cut the neocortex following the shortest geodesic path between the two poles. This artificial cut is robustly located as illustrated on Fig 2 .

We now suggest to decompose the parameterization of the neocortex into two steps:

- We first define a mapping $f: S_{T} \rightarrow \Omega$ from the surface of the neocortex onto a $2 \mathrm{D}$ rectangle, which corresponds to the integration of the constraints associated to the poles;

- and then include the sulcal constraints, which is thus reduced to a purely $2 \mathrm{D}$ deformation problem resulting in a second mapping $g: \Omega \rightarrow \Omega$.

The parameterization mapping $h$ is then defined as the composition of $f$ and $g$ : $h=g \circ f$. 


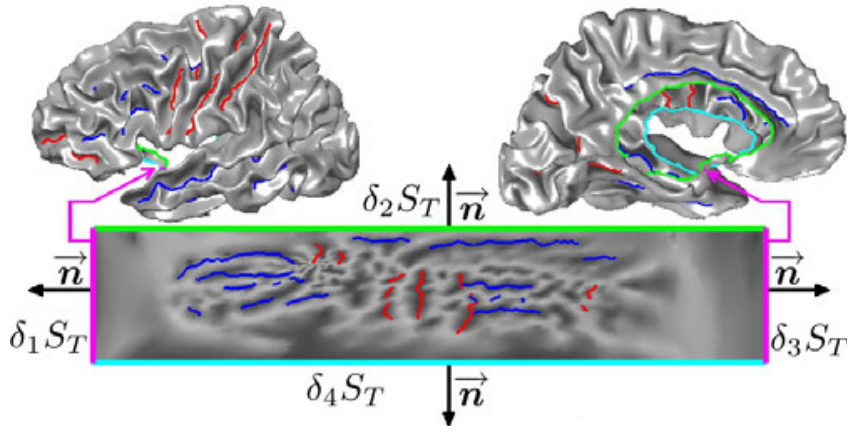

Fig. 2. Each cortical surface is mapped onto a rectangle. Boundaries and sulcal constraints are colored: latitude constraints in blue, longitude constraints in red, insular pole in cyan, cingular pole in green and cut between poles in pink. Mean curvature of the original surface is shown in gray and $\vec{n}$ is the normal of the boundary.

\subsection{Unconstrained Harmonic Mapping of the Neocortex onto a Rectangle}

Given the intrinsic orthogonal organization of the cortical surface [1]3 the mapping between the neocortex and the rectangular domain should minimize angular distortions. Such mapping has been intensively studied and is denoted as conformal or harmonic mapping. Several research groups have reported work on conformal mapping from the cortical surface to a sphere 11] possibly with additional sulcal landmarks [12]. Hurdal et al. 9] reported a discrete mapping approach that uses circle packing to produce flattened images of cortical surfaces on the sphere, the Euclidean plane, or the hyperbolic plane.

Here, we extend the method introduced to the computer graphics community by Eck et al. [6] which consists in approximating a harmonic map using a finite element method based on P1 basis functions. This technique consists in two steps.

1. First fix the boundary mapping, i.e. fix $f_{\delta S_{T}}=f^{0}$, by mapping the boundary $\delta S_{T}$ homeomorphically to some convex polygon in the plane. Here this parameterization domain is defined as a planar rectangle.

2. Find the piecewise linear mapping $f: S_{T} \rightarrow \Omega$ which minimizes the Dirichlet energy subject to the Dirichlet boundary condition $f_{\delta S_{T}}=f^{0}$ :

$$
E_{D}(f)=1 / 2 \int_{S_{T}}\left\|\nabla_{S_{T}} f\right\|^{2}
$$

Fixing the boundary remains however a major drawback in the context of our model: the conformality of the mapping is not guaranteed and the orthogonality of coordinate axis is lost near the boundary. We thus adapted this procedure by relaxing the boundary constraints such that the points on the boundary can move along the coordinate parallel to the boundary and only the four points in the corners remain fixed (see Fig 2 and Eq2). 
Derivating the Dirichlet energy $E_{D}(f)$ gives $\nabla E_{D}(f)=-\Delta_{S_{T}} f$ and its minimization reduces to solving independently the two linear system of equations which are Poisson equations with mixed boundary conditions:

$$
\left\{\begin{array} { l } 
{ \Delta _ { S _ { T } } f _ { 1 } = 0 , \in S _ { T } , } \\
{ f _ { 1 } = f _ { 1 } ^ { 0 } \text { on } \delta _ { 1 } S _ { T } \bigcup \delta _ { 3 } S _ { T } } \\
{ \nabla f _ { 1 } \cdot \vec { \boldsymbol { n } } = 0 \text { on } \delta _ { 2 } S _ { T } \bigcup \delta _ { 4 } S _ { T } }
\end{array} \quad \left\{\begin{array}{l}
\Delta_{S_{T}} f_{2}=0, \in S_{T} \\
\nabla f_{2} \cdot \overrightarrow{\boldsymbol{n}}=0 \text { on } \delta_{1} S_{T} \bigcup \delta_{3} S_{T} \\
f_{2}=f_{2}^{0} \text { on } \delta_{2} S_{T} \bigcup \delta_{4} S_{T}
\end{array}\right.\right.
$$

where $\delta S_{T}=\delta_{1} S_{T} \bigcup \delta_{2} S_{T} \bigcup \delta_{3} S_{T} \bigcup \delta_{4} S_{T}$ is the domain boundaries as shown on Fig 2, The existence and uniqueness of the solution has been proved (e.g. in [7]) and $\Delta_{S_{T}}$ can be discretized with the harmonic weights defined in [6. Inverting the two matrices involved in those two linear equations can be achieved in few seconds with efficient linear algebra libraries.

\subsection{Harmonic Mapping under Orthogonal Constraints}

We now define two sets of constraints $K_{\text {lat }}$ and $K_{\text {lon }}$ corresponding to the sulcal fundi specified in our anatomical model (as latitudes and longitudes respectively). Contrary to other methods, we want to align those sulcal fundi onto specific coordinates (longitude or latitude) and not to other landmarks.

The constrained mapping $g: \Omega \rightarrow \Omega$ that minimizes the angle distortion is defined as the optimum of the following equation under the same boundary conditions as in Eq,2.

$$
E(g)=E_{D}(g)+\lambda E_{P}(g)=\frac{1}{2} \int_{\Omega}\left\|\nabla_{S_{T}} g\right\|^{2}+\frac{\lambda}{2} \int_{\Omega}\|g(u)-p(g(u))\|^{2} d u
$$

where $p$ is defined as: $p:\left(\begin{array}{l}u_{1} \\ u_{2}\end{array}\right) \rightarrow\left(\begin{array}{l}u_{1}^{\text {lon }} \\ u_{2}\end{array}\right)$ if $u \in K_{\text {lon }}, \mathrm{p}:\left(\begin{array}{l}u_{1} \\ u_{2}\end{array}\right) \rightarrow\left(\begin{array}{l}u_{1} \\ u_{2}^{\text {lat }}\end{array}\right)$ if $u \in K_{\text {lat }}$, and $p=$ Id otherwise. $E_{P}(g)$ measures the distance between each sulcal fundus and the corresponding target longitude (resp. latitude) coordinate value $u_{1}^{\text {lon }}\left(\right.$ resp. $u_{2}^{\text {lat }}$ ) via the projection $p$. The derivative of the constraint energy $E_{P}(g)$ can be computed as:

$$
\nabla E_{P}(g)=2\left(I-D_{g}^{*} p\right)(g-p(g))
$$

where $D_{g}^{*} p$ is the adjoint operator applied to the derivative matrix of $p$, i.e.

$$
D_{g}^{*} p(u)=\left(\begin{array}{ll}
\frac{\partial p_{1}}{\partial u_{1}} & \frac{\partial p_{1}}{\partial u_{2}} \\
\frac{\partial p_{2}}{\partial u_{1}} & \frac{\partial p_{2}}{\partial u_{2}}
\end{array}\right)^{t}=\left(\begin{array}{ll}
1 & 0 \\
0 & 0
\end{array}\right) \text { if } u \in K_{l o n}, D_{g}^{*} p(u)=\left(\begin{array}{ll}
0 & 0 \\
0 & 1
\end{array}\right) \text { if } u \in K_{\text {lat }}
$$

and $D_{g}^{*} p=I d$ otherwise. We thus obtain

$$
\nabla E(g)=-\Delta_{S_{T}} g+\lambda\left(I-D_{g}^{*} p\right)(g-p(g))
$$

and the energy is then minimized using a classical iterative gradient descend scheme. Note that the projection of a point onto the corresponding axis $p(g(u))$ depends on its current location $g(u)$ which is updated between two iterations. This original data-driven term $E_{P}(g)$ introduces a coupling between the two coordinate fields, latitudes and longitudes. 


\section{Results}

We applied this parameterization process with $\lambda=1$ to the left and right cortical surfaces of 34 subjects, for which sulci were identified manually by an expert after an automatic extraction of cortical surfaces using the BrainVisa $11 \mathrm{~T} 1$ processing pipeline on T1-weighted MR images of the subjects. Fig 3 presents the mapping on the rectangular domain of all sulcal fundi that are part of the model for all 34 subjects. Top row shows the result of the unconstrained conformal mapping presented in section 2.2 and bottom row shows the results of the constrained mapping presented in section 2.3 . It is visible that the mapping without sulcal

\section{Left hemisphere Right hemisphere}

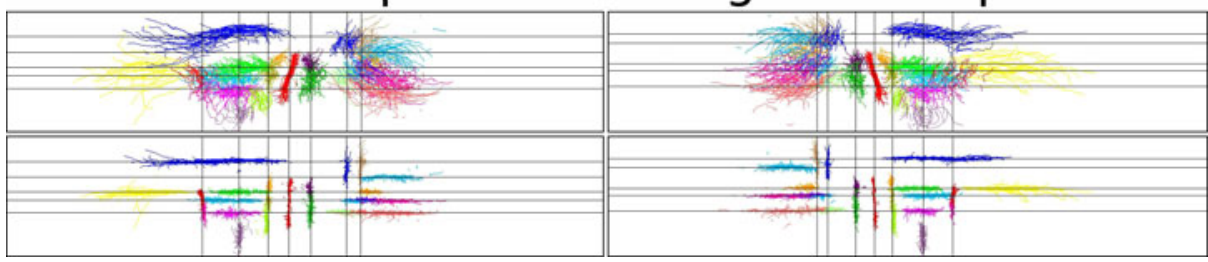

Fig. 3. Sulcal constraints of the 34 subjects mapped onto the rectangular domain with unconstrained mapping (top row) and with the model-driven mapping (bottom row). The sulci are colored according to the model shown on Fig 1

constraints shows a reasonable orthogonality of "longitude" and "latitude" sulci, which tends to advocate for the notions of orthogonal principal direction fields introduced in [13], and illustrates the pertinence of the model presented in [2]. On the bottom row of Fig 3 , the effects of the model-driven harmonic mapping are very clear. Sulci have been well aligned on the axis of the model. The implicit inter-subject matching performed by our method can also be observed since sulci show a good alignment across subjects. Residual variability is also visible, often at the extremity of sulcal fundi for which the assumption of alignment is sometime corrupted. It is also understood that this assumption is subject to variability and cannot be systematically observed due to the complexity of the folding process during growth, which is subject to many factors. The intersubject matching can also be checked on Fig 4 in which the mean curvature has been averaged across all subjects and is represented on a single cortical surface. The major sulcal and gyral structures are well preserved: deep blue areas indicate that the dispersion of sulci has been reduced, and red parts show that the crown of gyri are matched across subjects although they are not part of the model. The improved alignment of gyri shows that the coordinates are well interpolated between sulcal constraints and that the harmonic properties of the mapping help to align a number of gyri that are also subject to the orthogonal organization. The density of the model constraints also shows its influence for instance in

\footnotetext{
1 http://brainvisa.info
} 


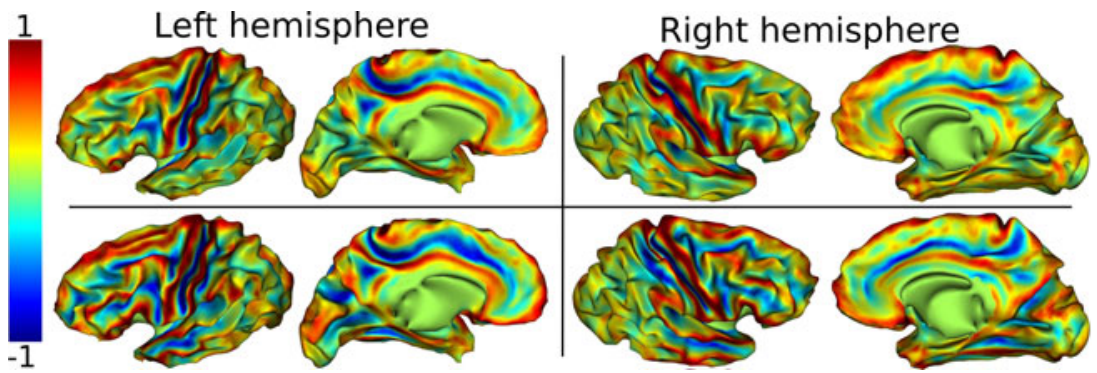

Fig. 4. Mean curvature averaged across 34 subjects. Top row: after unconstrained planar mapping; bottom row: after mapping constrained by the model.

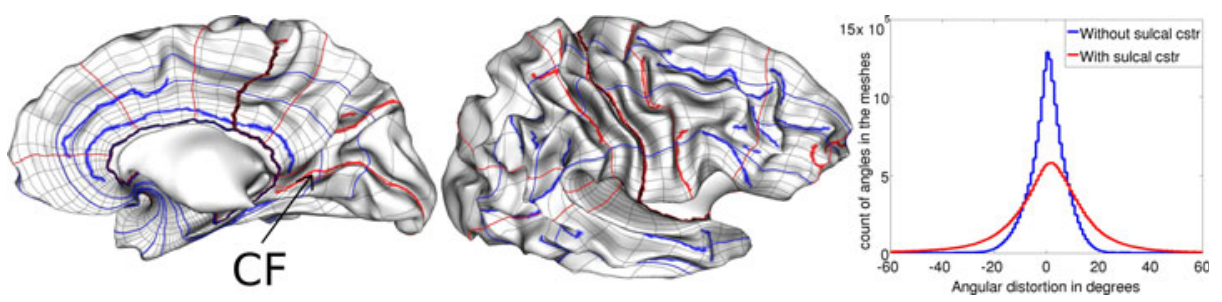

Fig. 5. The resulting parameterization, shown as a coordinate grid on the slightly smoothed cortical surface of one hemisphere. In blue (resp. red), the sulci and axes of the rectangular domain corresponding to latitude (resp. latitudes) constraints of the model. The histogram of the angular distortion through all 34 left and right cortical surfaces before (blue) and after (red) sulcal alignment is shown on the right.

the parietal lobe where the model lacks information [2] and the alignment of curvatures is not as good.

The adaptation of the resulting coordinate grid to local anatomy can be seen for one subject on Fig 5 . Iso-coordinate axes follow the local geometry and comply with the sulcal constraints. See e.g. the Calcarine Fissure (CF) which is a good predictor of the position of primary visual functional areas. Finally, Fig [5 also shows the control over angular distortions, as indicated by their distribution through the 34 left and right cortical surfaces before and after the constrained mapping.

\section{Conclusion}

We have proposed here an implementation of the cortical model presented in [2], performed via a mapping to a planar domain. The harmonic aspect of our mapping allows to explicitly take into account the directional nature of the model, based on the notion of orthogonality of sulcal axis. As opposed to previous harmonic approaches, we do not match folds across subjects or between subjects and a template, but instead we optimize the fit between cortical sulci and specific 
iso-coordinate axis in the model. Results show a good alignment of structures, sulci and gyri, across subjects. The process should prove useful for cortical localization and surface matching in the context of surface-based group analysis of functional and anatomical studies, although more work remains to be done, in particular to compare our method to reference methods such as [14].

\section{References}

1. Régis, J., Mangin, J.-F., Ochiai, T., Frouin, V., Rivière, D., Cachia, A., Tamura, M., Samson, Y.: Sulcal roots generic model: a hypothesis to overcome the variability of the human cortex folding patterns. Neurol. Med. Chir. 45, 1-17 (2005)

2. Clouchoux, C., Rivière, D., Mangin, J.-F., Operto, G., Régis, J., Coulon, O.: Modeldriven parameterization of the cortical surface for localization and inter-subject matching. NeuroImage 50, 552-566 (2010)

3. Toro, R., Burnod, Y.: Geometric atlas: modeling the cortex as an organized surface. NeuroImage 20, 1468-1484 (2003)

4. Lohmann, G., Von Cramon, Y., Colchester, A.: Deep sulcal landmarks provide an organizing framework for human cortical folding. Cereb. Cortex 18, 1415-1420 (2008)

5. Desbrun, M., Meyer, M., Alliez, P.: Intrinsic parameterizations of surface meshes. Computer Graphics Forum 21, 209-218 (2002)

6. Eck, M., DeRose, T., Duchamp, T., Hoppe, H., Lounsbery, M., Stuetzle, W.: Multiresolution analysis of arbitrary meshes. In: 22nd Conference on Computer Graphics and Interactive Techniques SIGGRAPH 1995, pp. 173-182. ACM, New York (1995)

7. Allaire, G.: Analyse numérique et optimisation. Éditions de l'École Polytechnique (2005)

8. Lui, L., Thiruvenkadam, S., Wang, Y., Thompson, P., Chan, T.: Optimized Conformal Surface Registration with Shape-based Landmark Matching. SIAM Journal on Imaging Sciences 3, 52-78 (2010)

9. Hurdal, M.K., Stephenson, K.: Discrete conformal methods for cortical brain flattening. Neuroimage 45, 86-98 (2009)

10. Joshi, A., Shattuck, D., Thompson, P., Leahy, R.: Surface-constrained volumetric brain registration using harmonic mappings. IEEE Trans. Med. Imag. 26, 16571669 (2007)

11. Haker, S., Angenent, S., Tannenbaum, A., Kikinis, R., Sapiro, G., Halle, M.: Conformal surface parameterization for texture mapping. IEEE Trans. Vis. Comput. Graphics 6, 181-189 (2000)

12. Wang, Y., Lui, L.M., Chan, T.F., Thompson, P.M.: Optimization of brain conformal mapping with landmarks. In: Duncan, J., Gerig, G. (eds.) MICCAI 2005. LNCS, vol. 3750, pp. 675-683. Springer, Heidelberg (2005)

13. Tosun, D., Rettmann, M.E., Prince, J.L.: Mapping techniques for aligning sulci across multiple brains. Med. Image Anal. 8, 295-309 (2004)

14. Fischl, B., Sereno, M.I., Tootell, R.B., Dale, A.M.: High-resolution intersubject averaging and a coordinate system for the cortical surface. Hum. Brain Mapp. 8, 272-284 (1999) 